\title{
The clinical value of CXCR4, HER2 and CD44 in human osteosarcoma: A pilot study
}

\author{
QIONG MA* , YONG ZHOU* ${ }^{*}$ BAOAN MA, XIANG CHEN, YANHUA WEN, \\ YUNYAN LIU, QINGYU FAN and XIUCHUN QIU \\ Orthopaedic Oncology Institute of Chinese PLA, Tangdu Hospital, \\ The Fourth Military Medical University, Xi'an 710038, P.R. China
}

Received September 10, 2011; Accepted December 13, 2011

DOI: $10.3892 / \mathrm{ol} .2012 .558$

\begin{abstract}
The biological markers CXCR4, HER2 and CD44 are involved in tumor growth and the homing of cancer cells to distant sites. The aim of this retrospective, case-control study was to evaluate whether the expression of CXCR4, HER2 and CD44 correlated with poor prognosis. Expression levels of CXCR4, HER2 and CD44 were evaluated by immunohistochemical staining in paraffin-embedded tissue sections of malignant primary osteosarcoma and related metastatic carcinoma from 63 patients, at a median follow-up of 5.5 years (range 1-10 years). Associations between these parameters and clinical features were examined. Of the 63 specimens, there were 38 HER2-positive, 57 CXCR4-positive and 62 CD44positive cases. There were 36 cases of HER 2 and CXCR 4 co-expression, 38 cases of HER 2 and CD44 co-expression and 56 cases of CXCR 4 and CD 44 co-expression. A total of 33 cases were CXCR4, HER2 and CD44 co-positive. Surface expression of CXCR4, HER2 and CD44 was high in the selected tissue samples, and CD44 was the most highly expressed. We observed a significant trend for a higher frequency of the expression of the three biomarkers in high-grade compared to low-grade osteosarcoma. Our results suggest a pivotal role of CXCR4, HER2 and CD44 expression as a prognostic factor of malignant human osteosarcoma. A larger study is ongoing to confirm these results.
\end{abstract}

\section{Introduction}

At present, the ability to predict prognosis at diagnosis in osteosarcoma is limited. The most significant predictor of outcome at diagnosis is the presence of metastases. However, $20 \%$ of patients who present at diagnosis with metastatic disease have

Correspondence to: Dr Xiuchun Qiu, Orthopaedic Oncology Institute of Chinese PLA, Tangdu Hospital, The Fourth Military Medical University, Xi'an 710038, P.R. China

E-mail: qiuxiuchun2009@live.cn

*Contributed equally

Key words: CXCR4, HER2, CD44, osteosarcoma an extremely poor prognosis. The metastases predictor is of no use to the remaining $80 \%$ of patients who present with local disease only. In these patients, a limited number of determinants that predict outcome have been identified. Some of the biological factors evaluated thus far as potential prognostic factors in osteosarcoma are the expression of CXCR4, human epidermal growth factor receptor-2 (HER2) and CD44.

CXCR4 expression on tumor cells is upregulated by hypoxia and angiogenic factors, including vascular endothelial growth factor (VEGF) (1). It is capable of directing the trafficking of normal and malignant cells to organs that express high levels of stromal cell-derived factor-1 (SDF-1), including the lymph nodes, lungs, liver and bone, as well as acting as a co-receptor for the entry of human immunodeficiency virus in CD4(+) T cells. More importantly, CXCR4 has been found to be a prognostic marker in various types of cancer, including leukemia and breast cancer, and recent evidence has highlighted the role of CXCR4 in prostate cancer (2).

The HER2 proto-oncogene (also known as HER2 neu or c-erbB-2) is located at 17q21 and encodes a $185-\mathrm{kDa}$ transmembrane tyrosine kinase glycoprotein with extensive homology to the epidermal growth factor receptor (3). Amplification of this gene with overexpression of the gene product occurs in approximately $30 \%$ of cases of breast cancer and is considered to be a poor prognostic indicator for this tumor. In certain other types of tumors, such as ovarian and lung cancer, enhanced expression of HER 2 has been associated with increased tumorigenicity and metastatic potential or with resistance to chemotherapeutic agents. In musculo-skeletal tumors, the expression of HER 2 at diagnosis has been analyzed in soft-tissue sarcoma (4,5), and among bone neoplasms in Ewing's sarcoma (6) and osteosarcoma (7). However, results for HER 2 expression in osteosarcoma are controversial, with some studies reporting up to $61 \%$ of positive cases and others reporting only negative results. Furthermore, the expression of HER 2 is reported to be an unfavorable prognostic indicator by some groups and a favorable one by others, one study even indicated a positive association between increased levels of HER2 and improved survival rates (8).

CD44 was previously thought to be a transmembrane adhesion molecule, which also played a role in the metabolism of its principal ligand hyaluronan. It may exist in three distinct physical phases, as a transmembrane cell surface receptor, 
an integral component of the matrix and in a fluid phase, each with the potential for being functionally significant. Investigations of CD44 over the past 20 years have established additional functions for CD44, including its capacity to mediate inflammatory cell function, tumor growth, adhesion, migration and metastasis. It has also become evident that intricate post-translational modifications of CD44 regulate the affinity of the receptor for its ligands (9).

One of the most significant predictors of outcome in osteosarcoma is the histological response of tumors to preoperative chemotherapy. The purpose of this study was to investigate whether the immunohistochemical detection of CXCR4, HER2 and CD44 protein at diagnosis may identify high-risk groups of osteosarcoma patients by predicting clinical outcome. Their contributions as biomarkers of tumor behavior as well as potential therapeutic targets may be confirmed by further studies.

\section{Materials and methods}

Samples.Atotal of 63 osteosarcoma samples were obtained from patients who underwent surgery at the Orthopaedic Oncology Center of Tangdu Hospital (China). Of the 63 patients, 33 were male and 30 were female (Table I). Patient ages ranged from 8 to 42 years (mean 16 years). The osteosarcomas were subclassified as osteoblastic $(n=38)$, chondroblastic $(n=2)$, fibroblastic $(n=4)$, telangiectatic $(n=2)$, small round cell tumor $(n=2)$ or mixed $(n=3)$. The specimens were obtained from the primary site in 51 patients and from the metastatic site in 12 patients. The location of the tumors were distal femur $(n=31)$, proximal tibia $(n=10)$, proximal humerus $(n=4)$, pelvis $(n=7)$, fibula $(n=1)$, thoracic vertebra $(n=2)$, thyroid $(n=1)$, lung $(n=1)$ and other locations $(n=6)$. All patients or their guardians provided written informed consent for participation in this study. The study was approved by the ethics committee of the hospital.

Methods. Patients received therapy according to the CCG 7921, Regimen A, with minor modifications. Induction chemotherapy with cisplatin, doxorubicin and high-dose methotrexate with leucovorin rescue preceded definitive surgical resection. Following recovery from definitive surgery, patients received maintenance chemotherapy with cisplatin, doxorubicin and high-dose methotrexate with leucovorin rescue. The chemotherapy efficacy was assessed histologically using the Huvos grading system. In this system, four grades have been described: Grade I, little or no evidence of necrosis; Grade II, tumor necrosis of 50-90\%; Grade III, necrosis between $90-99 \%$; and Grade IV, $100 \%$ necrosis. The chemotherapy responses were classified as Grade I $(n=29$, $46 \%)$, Grade II $(n=15,24 \%)$, Grade III $(n=12,20 \%)$ and Grade IV $(\mathrm{n}=7,10 \%)$. Tumor specimens were reviewed by a pathologist to ensure that there was $<30 \%$ contamination with normal stromal cells.

Immunohistochemistry. The protein expression of CXCR4, HER2 and CD44 was examined by immunohistochemistry of 4- $\mu \mathrm{m}$ sections using an avidin-biotin immunoperoxidase assay on optimal cutting temperature (OCT)-embedded frozen blocks. Sections were incubated with a primary antibody against CXCR4 (1:100 dilution; Santa Cruz Biotechnology,
Table I. Clinical characteristics of the bone tumor patients.

Characteristics

Number of cases $(\%)$

$\begin{array}{lc}\text { Gender } & \\ \text { Male } & 33(52) \\ \text { Female } & 30(48) \\ \text { Histological classification } & \\ \text { Osteoblastic } & 38(60) \\ \text { Chondroblastic } & 2(3) \\ \text { Fibroblastic } & 4(6) \\ \text { Telangiectatic } & 2(3) \\ \text { SRCT } & 2(3) \\ \text { Mixed } & 4(6)\end{array}$

Type of specimen

Primary

Metastasis

Primary site

Distal femur

Proximal tibia

10 (16)

Proximal humerus

Pelvis

7 (11)

Fibula

Thoracic vertebra

Thyroid

$1(1.4)$

Lung

Other

Metastasis at diagnosis

Present

Absent

Follow-up study

Amputee

Deceased

Survival

SRCT, small round cell tumor.

Santa Cruz, CA, USA), HER2 (1:100 dilution; Santa Cruz Biotechnology) and CD44 (1:200 dilution; Santa Cruz Biotechnology). Subsequently, the sections were incubated with biotinylated secondary antibodies, followed by avidinperoxidase, which formed a complex. Diaminobenzidine (1:50) was used as the final chromogen and hematoxylin was used as the nuclear counterstain. The intensity of immunostaining was scored as negative, low- , medium- and high-positive by a pathologist, blind to patient identity and clinical information. For each parameter, the immunostaining in patient samples was compared with normal bone tissue as a control.

Statistical analysis. The association of CXCR4, HER2 and CD44 protein expression with clinical data was investigated by the Chi square test. Follow-up data were used to analyze the relationship with survival. Differences between immunohistochemical staining results and clinical parameters were 
A

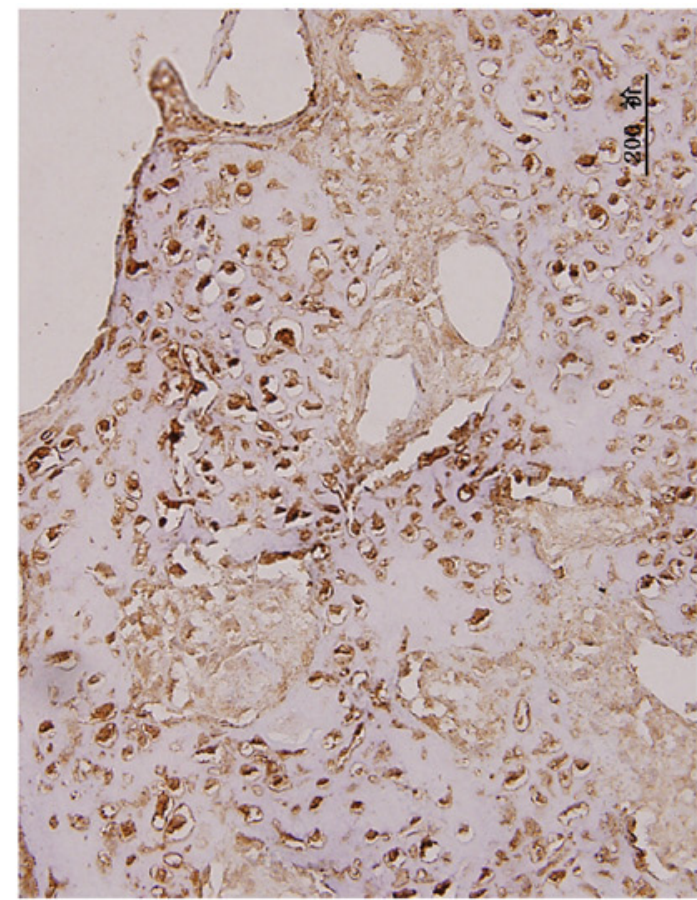

B

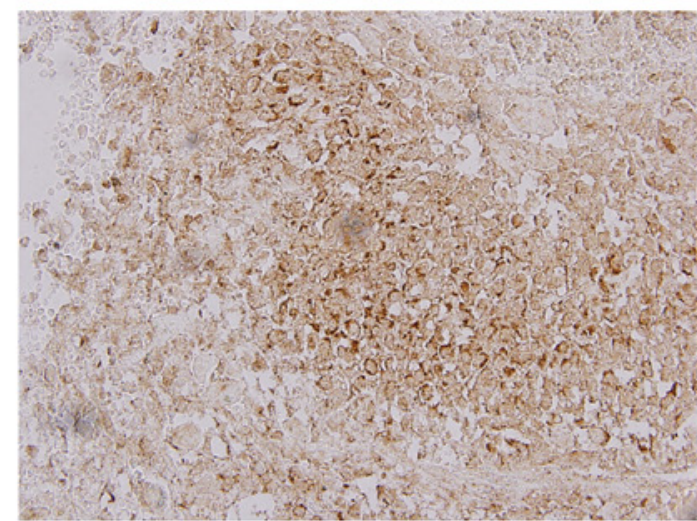

C

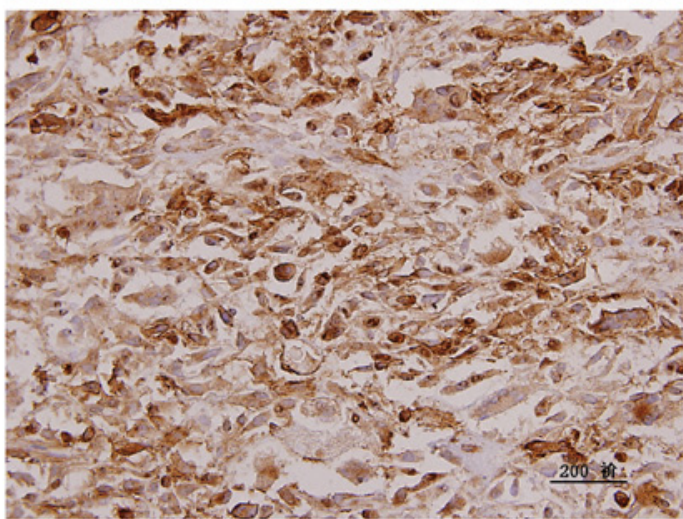

Figure 1. Representative immunohistochemical data showing osteosarcoma samples with high levels of CXCR4, HER2 and CD44 (original magnification, x400). (A) CXCR4; (B) HER2; (C) CD44.

compared using the Chi square test. An arbitrary p-value of 0.05 or less was considered to indicate a statistically significant difference.

\section{Results}

A total of 63 patients were entered into the study. Archival pathological material were identified from all the patients.
Table II. HER2 expression and metastatic disease.

\begin{tabular}{lcc}
\hline \multirow{2}{*}{ Metastatic disease } & \multicolumn{2}{c}{ Number of patients } \\
\cline { 2 - 3 } & HER2 (+) & HER2 (-) \\
\hline Yes & 12 & 0 \\
No & 26 & 25 \\
\hline
\end{tabular}

HER2, human epidermal growth factor receptor-2.

Table III. CXCR4 expression and metastatic disease.

\begin{tabular}{lcc}
\hline Metastatic disease & \multicolumn{2}{c}{ Number of patients } \\
\cline { 2 - 3 } & CXCR4 (+) & CXCR4 (-) \\
\hline Yes & 9 & 3 \\
No & 48 & 3 \\
\hline
\end{tabular}

Table IV. CD44 expression and metastatic disease.

\begin{tabular}{lcc}
\hline Metastatic disease & \multicolumn{2}{c}{ Number of patients } \\
\cline { 2 - 3 } & $\mathrm{CD} 44(+)$ & CD44 (-) \\
\hline Yes & 12 & 0 \\
No & 50 & 1 \\
\hline
\end{tabular}

The clinical characteristics recorded are outlined in Table I. CXCR4 staining was positive in 57 out of 63 patients (90.4\%), HER 2 staining was positive in 38 out of 63 patients $(60.3 \%)$ and CD44 staining was positive in 62 out of 63 patients (98.4\%). There were 36 patients with HER 2 and CXCR 4 co-expression, 38 patients with HER 2 and $\mathrm{CD} 44$ co-expression and 56 patients with CXCR4 and CD44 co-expression. In addition, there were 35 patients with CXCR4, HER2 and CD44 co-positive staining. Positive staining was predominantly restricted to the membrane and cytoplasm (Fig. 1). We observed an association between HER 2 and metastatic disease. Presence of metastases was related to HER2 protein expression. There were more $(\mathrm{p}=0.024)$ metastases in HER2-positive patients (12 out of 38 versus 0 out of 25, respectively) (Table II). However, there was no evidence to show that metastases were related to CXCR4 and CD44 ( $\mathrm{p}=5.152 ; \mathrm{p}=7.853$, respectively) (Tables III and IV). We also found that samples demonstrating CXCR4, HER2 and CD44 co-expression exhibited a higher aggressiveness according to the follow-up study; $71.4 \%$ of cases that were CXCR4, HER 2 and CD44 co-positive exhibited poor clinical outcome, were amputated or deceased (Table V).

\section{Discussion}

Osteosarcoma is also known as osteogenesis sarcoma, and its morbidity ranks second only to plasma cell myeloma. Approximately $20 \%$ of primary osteosarcomas metastasize, 
Table V. CXCR4, HER2 and CD44 expression and follow-up study.

\begin{tabular}{lcccc}
\hline Follow-up & \multicolumn{3}{c}{ Number of patients } \\
\cline { 2 - 5 } & CXCR4 (+) & HER2 $(+)$ & CD44 $(+)$ & All three markers $(+)$ \\
\hline Amputee & 5 & 10 & 14 & 9 \\
Deceased & 13 & 13 & 23 & 16 \\
Survival & 39 & 15 & 25 & 10 \\
\hline
\end{tabular}

and patients frequently succumb to the disease as a result of pulmonary metastases or diffused metastases. In recent years, the survival rate of patients suffering from osteosarcoma has markedly improved as the effectiveness of chemotherapy has progressed. However, the 5-year survival is only $55-65 \%$, and 2 -year survival in patients with pulmonary metastases is less than $25 \%$ (10-12). At present, there are no definite biomarkers at the early diagnosis stage. Thus, it is difficult for surgeons to decide what measures should be taken according to the malignancy. In all instances, there is not only one gene that contributes to the malignancy of tumors. In this study, we obtained samples from 63 high malignancy osteosarcoma patients. We determined the frequency of CXCR4, HER 2 and CD44 expression, and preliminarily determined whether these markers predicted the clinical outcome.

The major limitation of the present study is that it is an immunohistochemical analysis of an existing patient cohort. Although this group represents all material available from a single large institution, in any analysis of this type, the results should be considered exploratory. Any associations identified may not necessarily be causal. Caution should be taken in interpreting data. In particular, our primary clinical parameters being assessed were Huvos grade and metastases. Limited sample size severely curtails the power of the study. Negative results cannot be interpreted as a non-existent association, and the ability to analyze subgroups was limited. Despite these limitations, noteworthy results were obtained. Validation of these results in a subsequent prospective patient cohort may lead to the identification of predictive and prognostic factors, confirming the utility of reporting these results.

Our results reveal that CXCR4, HER2 and CD44 are positive in $90.4,60.3$ and $98.4 \%$ of the collected samples, respectively. As the samples selected in this study were all samples of highly malignant disease, the expression of CXCR4, HER2 and CD44 were much higher than those in other tumor specimens that were collected randomly (data not shown). Therefore, these data relate only to the characteristics of much more aggressive osteosarcomas, not an average level. We may speculate that aggressiveness is simultaneously related to the three molecules in osteosarcoma.

We also found an association between HER2 expression and metastatic disease. Of 12 metastatic samples, all 12 were HER2-positive. In bone tumors, several studies on HER2 have been performed for osteosarcoma and Ewing's sarcoma, the two most common types of neoplasms of the skeleton, but the findings reported thus far are contradictory (13-17). Particularly for osteosarcoma, the data reported are paradoxical, ranging from studies in which HER2-increased expression was detected in $63 \%$ of patients (14) to other studies in which it was uncommon or even absent $(13,15,17)$. Moreover, as extensively discussed by Anninga and colleagues (17), inconsistent findings have also been reported with regards to HER2 status and its prognostic significance. In certain studies, the increased levels of the HER 2 protein at diagnosis resulted in an association with poor histological response to chemotherapy and/ or a poorer clinical outcome (16), whereas other reports did not show any correlation between HER2 and poor treatment response or prognosis (14).

Discrepancies may have arisen from several sources, including differences in technical approach, tissue processing, antibodies used for the assay, result interpretation and treatment protocols. In this study, we assessed the expression of HER2 in homogeneously treated samples, the immunohistochemical detection was performed with extensively validated monoclonal antibodies, for which no cross-reactivity with other cellular antigens has been reported. Our results have shown that 38 cases out of 63 samples were HER2-positive (ranging from low- to high-positivity). Combined with the clinical characteristics, we found that all the metastatic specimens were HER2-positive, with statistical significance. This is of vital importance in early diagnosis and when administering individualized therapy.

The chemokine receptor CXCR4 is involved in tumor growth and the homing of cancer cells to distant sites (18), as well as angiogenesis (19) and cancer progression (20). In the present study, CXCR4 staining was positive in 57 out of 63 cases. However, there is no evidence to show that it is associated with metastases $(\mathrm{p}=5.152)$.

The adhesion molecule CD44 is often reported in lymphocytes, macrophages and mesenchymal stem cells. It participates in a wide variety of cellular functions including lymphocyte activation, recirculation and homing, hematopoiesis and tumor migration. Takahashi et al reported that the expression of CD44 on tumors has been described to, not only affect metastatic spread, but also tumor growth (21) and induction of oncogenes, including ras. In the samples we collected, CD44 was $98.4 \%$ positive in 63 patients, and the majority of these were strongly positive. We can thus deduce the relationship between CD44 and high malignancy. However, as with CXCR4, there is no evidence to correlate the significant relationship with metastatic disease in our cohort $(\mathrm{p}=7.853)$.

Among the CXCR4, HER2 and CD44 co-positive cases, there were 9 patients with amputation, 16 patients succumbed to the disease and only 10 survived, as the data of the 10 -year follow-up study revealed. A total of $71.4 \%(25 / 35)$ of the co-positive cases who presented with poor outcome underwent 
amputation or succumbed to the disease. Tumor progression is known to be a multi-factorial process. We speculated that the three genes may interact with each other, and that the effect of co-expression is far stronger than simply adding their individual effects together. Therefore, deletion of the three genes may effect tumor progression.

We examined CXCR4, HER2 and CD44 in highly malignant human osteosarcoma. HER2 is overexpressed in a substantial proportion of patients with metastases, confirming previous studies demonstrating this relationship in several other types of cancer. In particular, we have identified an adverse role of CXCR4, HER2 and CD44 co-expression in the progression of osteosarcoma according to the follow-up study, exhibiting higher aggressiveness and poor clinical outcome. Therefore, targeting CXCR4, HER2 and CD44 together has become a crucial area for cancer therapy research. Using small hairpin RNAs to target the expression of the three genes in human osteosarcoma may inhibit the growth and distribution of tumor cells, and even improve survival. This direction of investigation shows promise for the development of more efficient cancer therapies for osteosarcoma.

\section{Acknowledgements}

This study was supported by the National Natural Science Foundation of China (grant nos. 30873027 and 30973409).

\section{References}

1. Staller P, Sulitkova J, Lisztwan J, Moch H, Oakeley EJ and Krek W: Chemokine receptor CXCR4 downregulated by von Hippel-Lindau tumor suppressor pVHL. Nature 425: 307-311, 2003.

2. Liekens S, Schols D and Hatse S: CXCL12-CXCR4 axis in angiogenesis, metastasis and stem cell mobilization. Curr Pharm Des 16: 3903-3920, 2010.

3. Scotlandi K, Manara MC, Hattinger CM, Benini S, Perdichizzi S, Pasello M, Bacci G, Zanella L, Bertoni F, Picci P and Serra M: Prognostic and therapeutic relevance of HER 2 expression in osteosarcoma and Ewings sarcoma. Eur J Cancer 41: 1349-1361, 2005.

4. Foster H, Knox S, Ganti AK, et al: HER-2/neu overexpression detected by immunohistochemistry in soft tissue sarcomas. Am J Clin Oncol 26: 188-191, 2003.

5. Sato T, Peiper M, Heinecke A, et al: Expression of HER2/ neu does not correlate with survival in soft tissue sarcoma. Oncologie 26: 268-271, 2003.
6. Thomas DG, Giordano TJ, Sanders D, et al: Absence of HER2/ neu gene expression in osteosarcoma and skeletal Ewings sarcoma. Clin Cancer Res 8: 788-893, 2002.

7. Anninga JK, van de Vijver MJ, Cleton-Jansen AM, et al: Overexpression of the HER- 2 oncogene does not play a role in high-grade osteosarcomas. Eur J Cancer 40: 963-970, 2004.

8. Akatsuka T, Wada T, Kokai Y, et al: ErbB2 expression is correlated with increased survival of patients with osteosarcoma. Cancer 94: 1397-1404, 2002.

9. Cichy J and Puré E: The liberation of CD44. J Cell Biol 161: 839-843, 2003.

10. Broadhead ML, Clark JC, Myers DE, Dass CR and Choong PF: The molecular pathogenesis of osteosarcoma: a review. Sarcoma: 13 April, 2011 (E-pub ahead of print).

11. PosthumaDeBoer J, Witlox MA, Kaspers GJ and van Royen BJ: Molecular alterations as target for therapy in metastatic osteosarcoma: a review of literature. Clin Exp Metastasis 28: 493-503, 2011.

12. Savage SA and Mirabello L: Using epidemiology and genomics to understand osteosarcoma etiology. Sarcoma: 8 March, 2011 (E-pub ahead of print)

13. Kilpatrick SE, Geisinger KR, King TS, et al: Clinicopathologic analysis of HER-2/neu immunoexpression among various histologic subtypes and grades of osteosarcoma. Mod Pathol 14: 1277-1283, 2011.

14. Akatsuka T, Wada T, Kokai Y, et al: ErbB2 expression is correlated with increased survival of patients with osteosarcoma. Cancer 94: 1397-1404, 2002.

15. Maitra A, Wanzer D, Weinberg AG, et al: Amplification of the HER-2/neu oncogene is uncommon in pediatric osteosarcoma. Cancer 92: 677-683, 2001.

16. Zhou H, Randall RL, Brothman AR, et al: Her-2/neu expression in osteosarcoma increases risk of lung metastasis and can be associated with gene amplification. J Pediatr Hematol Oncol 25: 27-32, 2003.

17. Anninga JK, van de Vijver MJ, Cleton-Jansen AM, et al: Overexpression of the HER-2 oncogene does not play a role in high-grade osteosarcomas. Eur J Cancer 40: 963-970, 2004.

18. Sacanna E, Ibrahim T, Gaudio M, Mercatali L, Scarpi E, Zoli W, Serra P, Bravaccini S, Ricci R, Serra L and Amadori D: The role of CXCR4 in the prediction of bone metastases from breast cancer: a pilot study. Oncology 80: 225-231, 2011.

19. Liekens S, Schols D and Hatse S: CXCL12-CXCR4 axis in angiogenesis, metastasis and stem cell mobilization. Curr Pharm Des 16: 3903-3920, 2010

20. Sun X, Cheng G, Hao M, Zheng J, Zhou X, Zhang J, Taichman RS, Pienta KJ and Wang J: CXCL12/CXCR4/CXCR7 chemokine axis and cancer progression. Cancer Metastasis Rev 30: 269-270, 2011.

21. Takahashi K, Stamenkovic I, Cutler M, Saya H and Tanabe KK: CD44 hyaluronate binding influences growth kinetics and tumorigenicity of human colon carcinomas. Oncogene 11: 2223-2232, 1995 